\title{
Gestão do conhecimento: uma revisão crítica orientada pela abordagem da criação do conhecimento
}

Sergio Luis da Silva

Professor doutor do Departamento de Ciências da Informação e Programa de Pós-Graduação em Engenharia de Produção da Universidade Federal de São Carlos - UFSCar

E-mail:sergiol@power.ufscar.br

\section{Resumo}

Uma bem-sucedida sistematização da gestão do conhecimento deve considerar que o conhecimento pode existir em dois formatos, tanto na mente das pessoas, quanto em registros diversos; e a tecnologia da informação tem grande importância no acesso e na renovação dos conhecimentos. Seguindo essas preocupações, a essência da idéia de "criação do conhecimento" utilizada na área de gestão organizacional reside em pessoas poderem se encontrar e trocar experiências com outras pessoas que têm ou trabalham com certos tipos de conhecimentos, e a importância da tecnologia da informação é construir um suporte para que isso ocorra. Considerando esse ponto de vista, discutem-se, no presente artigo, esforços para trocas de conhecimentos, utilizando-se, para isso, o relacionamento entre dois formatos de conhecimentos - aqueles que são inerentes às habilidades pessoais (conhecimento tácito) e aqueles que são possíveis de verbalizar e registrar (conhecimento explícito) - em quatro tipos de conversões do conhecimento: socialização (tácito de um indivíduo para outro), externalização (explicitando partes do conhecimento tácito), combinação (conhecimento explícito de um indivíduo para o grupo) e internalização (captando no formato tácito o conhecimento explícito do grupo). Os argumentos aqui apresentados baseiam-se no fato de que uma efetiva criação e trabalho com o conhecimento apenas ocorre em um ambiente em que existe uma contínua conversão entre os dois formatos do conhecimento.

\section{Palavras-chave}

Gestão do conhecimento; Conhecimento tácito; Conhecimento explícito; Conversões do conhecimento.

\section{Knowledge management: a critical review based on the knowledge creation approach}

\begin{abstract}
Successful systematization of Knowledge Management has to take into consideration the following points: knowledge may exist in a personal and registered format; and information technology has great importance in the access and renewal of knowledge. Following this concern, the essence of the idea of "knowledge creation" used in organizational management area is that people can cope and exchange experiences with other people that have or work with some kind of knowledge. The importance of information technology is to build up a support for this to happen. Considering this point of view, efforts for exchange of knowledge are discussed, by using this relationship between two formats of knowledge - those which are inherent to personal abilities (tacit knowledge) and those which are possible to verbalize and register (explicit knowledge) - in four types of knowledge conversion: socialization (tacit from one individual to other), -externalization (explicit parts of tacit knowledge), -combination (explicit from one individual to the group), and -internalization (explicit from the group to individual tacit). Arguments are based on the fact that an effective creation of and work with knowledge can only exist in an environment where a continuous conversion between these two kinds of forms happens.
\end{abstract}

\section{Keywords}

Knowledge management; Tacit knowledge; Explicit knowledge; Knowledge conversion.

\begin{abstract}
Onde está a vida que perdemos vivendo? Onde está a sabedoria que perdemos no conhecimento?
\end{abstract} Onde está o conhecimento que perdemos na informação?

T.S. Eliot (The Rock, 1934)

\section{INTRODUÇÃO}

O conhecimento envolvido nas atividades organizacionais já tem sido abordado desde as primeiras teorias da administração, ao menos indiretamente, tanto pelas teorias da linha da administração dita "científica”, quanto pela linha das "relações humanas". Mesmo antes da revolução industrial e do advento dos estudos da administração, a forma de produção artesanal nas oficinas que produziam sob encomenda já fazia intenso uso da aprendizagem pela prática, por meio da transferência de conhecimentos entre mestres e aprendizes.

Nos anos 80, esse tema tornou-se mais presente com o advento das abordagens teóricas relacionadas à sociedade do conhecimento, ao aprendizado organizacional e às competências essenciais na gestão estratégica (Spender, 1996). Em anos recentes, as pesquisas nessas abordagens intensificaram suas aplicações práticas e o aprofundamento teórico, focalizando a necessidade de se entender como as organizações trabalham com o conhecimento para desenvolver novos produtos, novos processos e novas formas ou arranjos organizacionais mais flexíveis, proporcionando uma vantagem competitiva sustentável.

A implantação coordenada da Gestão do Conhecimento (GC) cria uma vantagem competitiva sustentável e de difícil imitação, pois está enraizada nas pessoas que trabalham na empresa, e não em recursos físicos, que são facilmente imitáveis pelos concorrentes (Quinn et alii, 1997) e menos flexíveis para reagir às incertezas do ambiente (Thomke \& Reinertsen, 1998).

O presente artigo tem como principal motivação aprofundar as discussões sobre GC apresentadas em artigo anterior (Silva, 2002), no qual foi exposta uma visão coesa e integrada da gestão do conhecimento com os processos empresariais da organização, em sintonia 
com suas estratégias para a competitividade. No artigo que se desdobra a seguir, ampliam-se e ao mesmo tempo aprofundam-se as discussões sobre o lado operacional da gestão do conhecimento. Especificamente, centra-se nos formatos e nas quatro conversões do conhecimento, da abordagem teórica da criação do conhecimento, que ao mesmo tempo em que é tão comentada e repetida na literatura, normalmente é pouco explicada em mais detalhes, de forma que possa ser efetivamente aplicada em modelos de gestão para trocas de conhecimentos na empresa.

\section{Parâmetros para a inserção da gestão do conhecimento na empresa}

Trabalhar com o conhecimento de forma coesa e integrada aos processos de negócios da empresa deve compreender um conjunto de diretrizes e recomendações básicas, fortemente inter-relacionadas e válidas para qualquer abordagem de GC, que foram sintetizadas em Silva (2002).

Essas diretrizes e recomendações básicas influenciam diretamente a riqueza do mercado de conhecimentos de uma empresa, que pode ser avaliado por abordagens que buscam mensurar estrategicamente a aprendizagem e o conhecimento. Duas das mais disseminadas dessas abordagens são as seguintes:

- a proposta do capital intelectual (Edvinsson \& Malone, 1998), que é a principal tentativa de avaliar os recursos não-tangíveis da empresa, envolvendo marcas e patentes, valores respeitados pela sociedade e também o conhecimento e a capacidade de aprendizado que as pessoas de uma empresa potencialmente possuem;

- e, parcial ou indiretamente, o balanced scorecard (Kaplan \& Norton, 1997), em uma parte de sua abordagem teórica de mensuração estratégica da empresa, quando se preocupa com medições da capacidade de aprendizagem da empresa, em correlação com seus esforços estratégicos.

Tais abordagens trabalham basicamente com os seguintes indicadores da capacidade da organização para realizar a GC (e a aprendizagem organizacional) de alto desempenho (Lethbridge, 1998):

- parâmetros organizacionais (por exemplo, disseminação do trabalho em times, rotatividade entre diferentes postos de trabalho etc.);

- parâmetros de recursos humanos (por exemplo, gerenciamento de competências, programas de treinamento e formação de pessoas etc.);
- e parâmetros de sistemas de informação (por exemplo, a existência e disseminação da intranet/internet, de ferramentas de trabalho em grupo virtual etc.).

A mensuração estratégica da aprendizagem e do conhecimento em uma empresa está diretamente relacionada à disseminação e incorporação das já citadas diretrizes e recomendações básicas pela empresa, particularmente em suas áreas e departamentos funcionais. Orientado pela visão coesa e integrada dos processos de negócios, nota-se cada vez mais um alargamento ou flexibilização das atividades das áreas e departamentos funcionais da empresa, para assim contribuír com a GC e a aprendizagem organizacional nos processos de negócio em que estão envolvidas (Amidon, 1997). Exemplos dessas novas atribuições em algumas áreas funcionais são relacionadas em Silva (2002).

\section{Diferenciação do conhecimento em relação a dados e informações}

Particularmente importante para se entender a GC e, em especial, a teoria da criação do conhecimento, é analisar as discussões referentes às diferenças entre dados, informação e conhecimento. Há vários autores que buscam destacar a diferença existente entre dados, informação e conhecimento (Dutta, 1997; Marshall, 1997; Davenport \& Prusak, 1998), porém não existe propriamente um consenso quanto à diferenciação ou definição entre esses três conceitos.

Segundo Tuomi (1999), normalmente tratam-se esses conceitos em um sentido hierárquico, em que os dados são simples fatos que se tornam informação, se forem combinados em uma estrutura compreensível; ao passo que a informação torna-se conhecimento, se for colocada em um contexto, podendo ser usada para fazer previsões. Uma informação é convertida em conhecimento quando um indivíduo consegue ligá-la a outras informações, avaliando-a e entendendo seu significado no interior de um contexto específico.

De acordo com esse sentido, os dados são pré-requisitos para a informação, e esta é pré-requisito para o conhecimento. Tuomi (1999) menciona que uma hierarquia reversa a esta também faz sentido; nesse caso, a informação emerge somente após existir o conhecimento que permita compreender a sua estrutura, e os dados são percebidos somente após a informação que permite verificar a existência dos fatos.

As diversas definições dadas para o conhecimento normalmente consideram essas diferenciações 
hierárquicas e, em sua maioria, convergem para a idéia de que conhecimento é formado por informação, que pode ser expressa, verbalizada, e é relativamente estável ou estática, em completo relacionamento com uma característica mais subjetiva e não palpável, que está na mente das pessoas e é relativamente instável ou dinâmica, e que envolve experiência, contexto, interpretação e reflexão (Polanyi, 1966; Nonaka \& Takeuchi, 1997).

Essas duas partes constituintes do conhecimento são, na verdade, tratadas por muitos pesquisadores, entre eles Nonaka \& Takeuchi (1997), como dois tipos de conhecimentos intrinsecamente relacionados, quais sejam:

- o formato tácito, conhecimento subjetivo; habilidades inerentes a uma pessoa; sistema de idéias, percepção e experiência; difícil de ser formalizado, transferido ou explicado a outra pessoa;

- o formato explícito, conhecimento relativamente fácil de codificar, transferir e reutilizar; formalizado em textos, gráficos, tabelas, figuras, desenhos, esquemas, diagramas, etc., facilmente organizados em bases de dados e em publicações em geral, tanto em papel quanto em formato eletrônico.

Os formatos tácito e explícito do conhecimento podem ser bem entendidos e diferenciados pela analogia que Bond \& Otterson (1998) fazem com o trabalho de um artesão - escultor de madeira* - e que Mascitelli (2000) ilustra com a diferenciação das habilidades entre dois pianistas - músicos $^{* *}$.

As conversões do conhecimento entre estes dois formatos constitui a essência da abordagem teórica da criação do conhecimento, que será detalhada na próxima seção.

\footnotetext{
* Esse escultor experiente pode escrever detalhadas regras e procedimentos e construir elaboradas ferramentas físicas, porém, consegue incorporar nestes recursos formais apenas parte de seu conhecimento, aqueles que o escultor consegue externalizar na forma explícita. Mas quando este inicia uma nova escultura, um dos mais importantes conhecimentos é a visão do todo, do resultado final de seu trabalho, que o vai guiar nos detalhes e pequenas tarefas para conseguir seu intento. Esse conhecimento é tácito, apenas precariamente externalizado na forma de um desenho ou discurso, mas pode ser razoavelmente bem captado e incorporado por um aprendiz deste artesão que junto com ele trabalha por um regular intervalo de tempo.

** Ao descrever que dois pianistas, um aprendiz e um mestre, podem ter acesso ao mesmo conhecimento explícito (as partituras musicais), porém, o entendimento destas partituras será diferente, assim como a reação a esta leitura, em termos de interação com o teclado e ajuste ao som produzido (conhecimento tácito).
}

\section{Criação do conhecimento: formatos e conversões}

Os autores mais relevantes da abordagem teórica da criação do conhecimento e, portanto, da definição e do uso dos formatos tácito e explícito, Nonaka \& Takeuchi (1997), consideram que um trabalho efetivo com o conhecimento somente é possível em um ambiente em que possa ocorrer a contínua conversão entre esses dois formatos.

Esse foco na criação de conhecimentos por meio de espaços para a contínua conversão entre o formato tácito e explícito forma o núcleo central de uma das principais abordagens sobre GC, desenvolvida pelo trabalho dos pesquisadores Ikuhiro Nonaka e Hirotaka Takeuchi (Nonaka \& Takeuchi, 1997; Nonaka \& Konno, 1998), que detalharam esta conversão de conhecimentos entre os formatos tácito / explícito em quatro modos.

Uma ou mais conversões do conhecimento podem ocorrer simultaneamente. Os quatro modos existentes estão detalhados em seguida, em termos de ações em que a conversão entre o formato tácito-explícito do conhecimento normalmente ocorre.

\section{Socialização}

Conversão de parte do conhecimento tácito de uma pessoa no conhecimento tácito de outra pessoa. Esse tipo de conversão também é abordado pelas teorias ligadas à cultura organizacional e ao trabalho em grupo. Normalmente, esse conhecimento compartilhado acontece quando:

- ocorre diálogo freqüente e comunicação "face a face";

- brainstorming, insights e intuições são valorizados, disseminados e analisados (discutidos) sob várias perspectivas (por grupos heterogêneos);

- valoriza-se o trabalho do tipo "mestre-aprendiz": observação, imitação e prática acompanhada por um tutor;

- há compartilhamento de experiências e modelos mentais via trabalho em equipe.

Uma frase síntese dessa conversão pode ser, então, "troca de conhecimentos face a face entre pessoas".

\section{Externalização}

Conversão de parte do conhecimento tácito do indivíduo em algum tipo de conhecimento explícito. Esse tipo de conversão é pouco abordado por outras teorias da administração. Normalmente, esse conhecimento conceitual acontece por meio de: 
- representação simbólica do conhecimento tácito através de modelos, conceitos, hipóteses etc., construídos por meio de metáforas/analogias ou dedução/indução, fazendo uso de toda a riqueza da linguagem figurada para tentar externalizar a maior fração possível do conhecimento tácito;

- descrição de parte do conhecimento tácito, por meio de planilhas, textos, imagens, figuras, regras (por exemplo, nos sistemas especialistas), scripts, design history etc.;

- relatos orais e filmes (gravação de relatos orais e imagens de ocorrências/ações).

Uma frase síntese dessa conversão pode ser, então, "o registro do conhecimento da pessoa feito por ela mesma".

\section{Combinação}

Conversão de algum tipo de conhecimento explícito gerado por um indivíduo para agregá-lo ao conhecimento explícito da organização. Esse tipo de conversão também é abordado pelas teorias ligadas ao processamento da informação. Normalmente, esse conhecimento sistêmico acontece por meio do agrupamento (classificação, sumarização) e processamento de diferentes conhecimentos explícitos.

Uma frase síntese desta conversão pode ser, então: o agrupamento dos registros de conhecimentos.

\section{Internalização}

Conversão de partes do conhecimento explícito da organização em conhecimento tácito do indivíduo. Esse tipo de conversão também é abordado pelas teorias ligadas à aprendizagem organizacional. Normalmente, esse conhecimento operacional acontece por meio de:

- leitura/visualização e estudo individual de documentos de diferentes formatos/tipos (textos, imagens etc.);

- prática individual (learning by doing);

- reinterpretar/reexperimentar, individualmente, vivências e práticas (practices e lessons learned).

Uma frase síntese desta conversão pode ser, então: “o aprendizado pessoal a partir da consulta dos registros de conhecimentos".

Os ciclos de conversão do conhecimento, passando várias vezes por esses quatro modos, formam uma espiral que serve para analisar e entender os mais diversos casos de criação e disseminação do conhecimento, sendo que cada caso terá suas particularidades ou especificidades.
A abordagem teórica da criação do conhecimento é uma contribuição relevante e ponto de partida para muitos outros trabalhos. As duas próximas seções destacam alguns importantes facilitadores que permitem otimizar o trabalho com o conhecimento tácito e com o conhecimento explícito nas conversões do conhecimento em que esses formatos fazem parte. Esses facilitadores podem ter apoio de algumas TI aplicadas direta ou indiretamente na GC, o que é discutido em uma seção posterior.

\section{Facilitadores do trabalho com o formato tácito do conhecimento}

Um dos principais facilitadores para o trabalho com esse formato do conhecimento são as redes de trabalho que ligam pessoas experientes e preparadas para atuar em grupo, interagindo basicamente por meio de ampla troca de conhecimentos tácitos.

A importância das redes de trabalho entre as pessoas em uma organização já foi apontada pelos resultados obtidos no pioneiro estudo de Allen (1977), confirmados por pesquisas mais recentes (Mcdermott, 1999; Gupta \& Govindarajan, 2000; Storck \& Hill, 2000), evidenciando seu papel na troca de conhecimentos (principalmente tácitos)*. Essas redes, também denominadas comunidades de prática, normalmente espontâneas e informais em relação à estrutura formal da organização, podem envolver pessoas de dentro e de fora da empresa na troca de experiências e na busca de novas abordagens para problemas comuns, continuando a existir conforme seus membros se identifiquem com o propósito do grupo (Lievrouw \& Finn, 1996; Wenpin, 2000).

Outro importante facilitador de conversões entre conhecimentos tácitos é a capacidade criativa (e de inovação) existente na empresa ligada à presença de talentos individuais e de motivação fornecida pelo ambiente organizacional, para a proposição de idéias e soluções originais, além do estímulo ao compartilhamento de experiências individuais no

\footnotetext{
* Em síntese, esses autores apontam algumas condições para o funcionamento dessas redes de trabalho: grupos e redes são voluntários e espontâneos, a empresa não deve formalizá-los ou forçar sua criação, mas apenas remover barreiras e motivar a participação; a empresa deve respeitar as lideranças e elementos de ligação (gatekeepers) que surgem nesses grupos ou comunidades, escolhidos não por sua posição na hierarquia formal da empresa, mas sim por suas habilidades (motivadoras e inovadoras) de condução do trabalho do grupo; a empresa deve respeitar e valorizar os termos e linguagens comuns que esses grupos adotam e proteger os canais de comunicação (físicos e virtuais) e as oportunidades para o compartilhamento de experiências criados por esses grupos.
} 
Gestão do Conhecimento: uma revisão crítica orientada pela abordagem da criação do conhecimento

ambiente de trabalho. Destacam-se nessa linha teórica diversos autores, como Senker (1995), Cummings \& Oldham (1997), Bond \& Otterson (1998), Ghoshal \& Nahapiet (1998).

Por fim, um significativo facilitador é a capacidade de aprendizagem individual e organizacional. Garvin (1993) destaca que uma organização de aprendizagem é aquela em que as pessoas envolvidas estão capacitadas ou têm habilidades para criar (externalizar), adquirir (internalizar) e disseminar (socializar) conhecimentos, assim como modificar comportamentos a partir da reflexão sobre estes conhecimentos. Tal reflexão que modifica comportamentos requer que a aprendizagem não seja somente adaptativa e cumulativa (denominada aprendizagem de ciclo único), mas também inovadora (aprendizagem de ciclo duplo) (Argyris \& Schon, 1978).

\section{Facilitadores do trabalho com o formato explícito do conhecimento}

A memória organizacional ou corporativa compõe-se de idéias criativas, da análise de falhas e sucessos, das experiências diárias etc. (Heijst et alii, 1997), cujos fundamentos, habilidades e princípios mantêm-se e são transmitidos principalmente por meio de conhecimentos tácitos (Moorman \& Miner, 1998; Cross \& Baird, 2000), sendo que podem ser parcialmente registrados e, portanto, convertidos em conhecimentos explícitos os procedimentos, regras e recomendações resultantes desta memória (Soltero, 1997).

Portanto, um dos facilitadores para o trabalho com o formato explícito do conhecimento é a capacidade de construção de lessons learned (a parte da memória organizacional que contém apenas o conhecimento explícito), significando registrar objetivamente uma determinada vivência, os erros levantados e as soluções adotadas.

Algumas recomendações devem ser seguidas na construção de learning history (termo sinônimo, assim como best/bad practices, do termo lessons learned)*, já que não se trata de uma simples redação de um relatório operacional. Dentre outros cuidados apontados por Kleiner \& Roth (1997) e Birchall \& Smith (1998), destacam-se a ênfase na síntese (texto enxuto), a redação impessoal, porém com comentários dos fatos relatados

\footnotetext{
* Outras denominações e procedimentos similares: relatos na forma de case method, scripts (papéis e cenas que descrevem uma seqüência de eventos), redes semânticas (relações hierárquicas entre objetos), frames (uma estrutura que inclui todo o conhecimento de um objeto em particular), mapas e/ou cenários, conforme indicados por Heijst et alii (1997), Soltero (1997), Birchall \& Smith (1998), entre outros.
}

(mostrando diferentes pontos de vista e contextualizando a ocorrência relatada), e os critérios de aprovação e acesso às lessons learned para os diferentes níveis e setores da empresa.

Se as recomendações anteriores estão mais preocupadas com a otimização da externalização (redação) e internalização (facilidade de leitura do que foi redigido), um outro facilitador importante para o trabalho com o formato explícito é a preocupação com as formas de melhor agrupar ou organizar estes conhecimentos (combinação).

Heijst et alii (1997) e Maurer (1998) destacam a importância de que, para cada conhecimento (explícito) que faça parte da memória corporativa, tenham-se bem claro seus atributos ou metaconhecimentos, auxiliando tanto que esse seja encontrado em um diretório, como também servindo para dar o contexto (particularidades da ocorrência) que originou o conhecimento explícito, facilitando seu entendimento e reutilização em outros contextos (Tschaitschian et alii, 1997):

- qual(is) o(s) processo(s) de negócios e produtos/serviços ao(s) qual(is) o conhecimento está relacionado;

- como está classificado em um domínio (em qual taxionomia/vocabulário/palavra chave se insere e como está inserido, quem são os autores e usuários mais frequentes do conhecimento);

- como está representado (forma de apresentação do conhecimento: meio papel, meio eletrônico, texto, desenho etc.), onde se localiza e como se classifica no tempo (por exemplo de registro, uso, atualização, etc, do conhecimento).

Os recursos de Tecnologia da Informação (TI) podem otimizar ainda mais a externalização, internalização e combinação do conhecimento explícito, quando partem de uma situação em que as recomendações e preocupações anteriormente expostas já são levadas em consideração. A próxima seção aborda as contribuições da TI para a GC, particularmente no formato do conhecimento em que faz sentido aplicar essa tecnologia: o explícito.

\section{Aplicações da tecnologia da informação na gestão do conhecimento}

A TI não resolve todos os problemas do trabalho com o conhecimento explícito, porém seu uso e suas potencialidades contribuem no encaminhamento de significativa parte da solução desses problemas. 
A TI é fundamental para a combinação (agrupamento) dos conhecimentos explícitos, mas não contribui significativamente com o formato tácito do conhecimento. Basicamente, o máximo que pode fazer para a troca de conhecimento tácito-tácito é facilitar que pessoas sejam encontradas (contactadas) (e a partir daí podendo ocorrer a socialização). No entanto, a TI pode facilitar as outras duas conversões do conhecimento, quando o formato tácito está em equilíbrio com o formato explícito. Assim sendo, pode facilitar a externalização (auxilia no registro do conhecimento) e a internalização (agiliza o acesso ao conhecimento explícito).

Os recursos de TI facilitam o trabalho em rede, podendo manter os conhecimentos descentralizados junto aos locais em que são mais gerados e/ou utilizados (Davenport et alii, 1998) e melhorando o grau de interatividade do usuário com os registros de conhecimentos (por exemplo com as lessons learned) (Davenport \& Prusak, 1998). A TI é efetivamente útil para a GC, se for empregada utilizandose uma sistemática interferência (interatividade) humana (Davenport et alii, 2001).

O emprego de sistemas (tecnologias) de informação na empresa deve ser condicionado às definições e escolhas da estrutura organizacional, e não o contrário, cabendo a esses sistemas o papel de facilitadores na existência dos espaços organizacionais voltados ao processamento dos aspectos cognitivos (Salerno, 1998), contribuindo com o tratamento e transmissão do conhecimento (explícito) (Mcdermott, 1999).

As tecnologias da informação pioneiras no trabalho com o conhecimento

Pode-se considerar que a utilização da TI para a gestão do conhecimento tem seus primórdios nos anos 70, quando essa passa de um foco voltado ao processamento de dados para um foco voltado à informação, exemplificado pela criação dos sistemas de suporte à decisão gerencial (DSS - decision support system) e nos sistemas de informação gerencial (MIS - management information system).

Nos anos 80, a evolução se direciona para os sistemas da informação baseados no conhecimento (KBS knowledge-based information systems), em que talvez sua face mais visível e conhecida sejam os sistemas especialistas (expert systems) (Dutta, 1997).

Esses sistemas desdobraram-se em inúmeras linhas de atuação, indo desde a utilização de sistemas baseados em inteligência artificial e modelos matemáticos e estatísticos para criar o conhecimento a partir do cruzamento de dados e informações presentes em bases de dados (Davenport \& Klahr, 1998) (p.ex.: data mining, data warehousing), até a representação do conhecimento em sistemas especialistas e redes neurais que procuram automatizar a tomada de decisões (Speel \& Aben, 1998; Milton et alii, 1999). Também os sistemas KBS podem melhorar a dinamicidade (facilidade de registro e sua manutenção por meio do uso de regras e modelos) da representação do conhecimento explícito das vivências registradas (lessons learned ou best/bad practices) (Liebowitz et alii, 1998; Vriens \& Hendriks, 1999).

A colaboração desses sistemas restringe-se ao apoio (recurso facilitador) para que uma pessoa registre (conhecimento explícito) o máximo possível de seu conhecimento tácito (externalização) e agrupe e processe esse registro de forma efetiva junto com outros registros de conhecimentos explícitos da organização (combinação) (Heijst et alii, 1997; Cross \& Baird, 2000).

A internalização e a socialização também são conversões importantes para a formação da memória organizacional. A primeira pode ser em parte ajudada pela TI (tanto recursos KBS, como Intranets/Internet que são mostradas na próxima subseção), mas é mais influenciada pelas redes e comunidades de prática. A segunda praticamente independe da TI, ocorre apenas se houver um trabalho face a face, seja em equipes formais, ou em redes e comunidades informais.

\section{A evolução no trabalho com o conhecimento através dos recursos da internet}

Dos anos 90 até os dias atuais, a evolução e a disseminação da internet (e intranets) têm concentrado as principais aplicações da TI para a gestão do conhecimento (Dieng, 2000). Esta evolução incorpora e integra alguns tipos de KBS, trazendo-lhes novas funcionalidades, como, por exemplo, os recursos multimídia e hipertexto, facilitando sua interatividade tanto para uso individual quanto em grupo (Marshall, 1997; Birchall \& Smith, 1998; Carayannis, 1998). Exemplos dessa integração são as ferramentas de apoio de trabalho em grupo suportadas por mecanismos de gerenciamento de documentos eletrônicos, ferramentas de navegação inteligente na internet, etc (Ruggles III, 1997).

Os recursos da intranet/internet facilitam o acesso aos diferentes conhecimentos explícitos acumulados na corporação, podendo mesmo personalizar seu uso de acordo com as preferências e necessidades de cada pessoa (Maurer, 1998). Permitem ainda que se façam comentários ou que se criem grupos de discussão virtuais (groupware forum) sobre esses conhecimentos e outros assuntos, 
Gestão do Conhecimento: uma revisão crítica orientada pela abordagem da criação do conhecimento

facilitando a externalização de experiências e opiniões, envolvendo grupos restritos, grupos amplos dentro da empresa ou mesmo parceiros e colaboradores externos à empresa (como clientes, fornecedores, consultores e especialistas de universidades e outros. (Klooster et alii, 1997; Maurer, 1998; Bolisani \& Scarso, 2000). Esses recursos também ganham cada vez mais espaço como ferramenta de treinamento virtual das pessoas na empresa, devido ao baixo custo envolvido e à rapidez em conectar várias pessoas em pontos remotos ou diferentes fábricas da corporação.

A utilização de TI focalizada na internet/intranets para a GC representa também a adoção de uma tecnologia base de padrões abertos e universais, o que facilita a integração com outros sistemas internos ou externos à empresa, resultando em uma tendência recente que é a formação de portais com o objetivo de centralizar o acesso à intranet da empresa e a sites relacionados ou de interesse da empresa na internet (Agosta, 1999; Koulopoulos \& Reynolds, 1999).

A internet/intranet possui uma filosofia diferente da maioria dos sistemas de informação tradicionais. Segundo Scott (1998), apresenta características favoráveis à criação do conhecimento, que são autonomia, redundância, caos criativo e variedade, em consonância com a abordagem da mudança constante dos conhecimentos entre o formato tácito e explícito (escola da criação do conhecimento). As listas de discussão facilitam o diálogo e a interação, os gráficos aumentam o uso de metáforas, analogias e protótipos para clarear o que estava originalmente confuso e obscuro. Hyperlinks relacionam conceitos e organizam os repositórios de conhecimentos para melhor acesso e trabalho cognitivo. A integração do conhecimento organizacional é facilitada pela capacidade de trabalhar com plataformas diferentes e padrões abertos (Scott, 1998).

Perspectivas recentes e síntese do uso da tecnologia da informação para a gestão do conhecimento

Paralelamente à evolução recente da internet e dos portais corporativos, uma TI que também pode influenciar na GC são os sistemas ERP (Enterprise Resource Planning), empregados pelas grandes empresas desde os anos 90.

Buscando integrar vários setores da empresa, os sistemas ERP procuram padronizar e normalizar os diferentes setores e funções, facilitando o compartilhamento de dados, informação e até conhecimentos (Davenport \& Prusak, 1998). Esses sistemas estruturam-se em torno de uma grande base de dados central que envolve toda a corporação (Davenport, 1998). Passa a ser uma ferramenta com impacto na GC à medida que tem como foco intermediar a ação de pessoas, aproximando quem domina determinados conhecimentos de quem os está necessitando. Com a nova utilização destes sistemas ERP por meio de intranet / internet (SAP, 2000) e integrados aos recentes sistemas CRM (Customer Relationship Management) que tratam de informações sobre clientes, ainda mais possibilidades de intermediação de conhecimentos podem ocorrer.

As intranets corporativas, bem como outros sistemas além do ERP, podem permitir o gerenciamento dos conteúdos de conhecimentos da empresa. Porém, uma importante parte da solução, para que estes sistemas funcionem, passa por se estabelecer muito bem quais são os processos-chave e os principais papéis e fluxos de trabalho (workflow) dentro desses processos (Elliott, 1999).

Sintetizando essa evolução histórica pela interpretação do trabalho de vários autores (Wiig, 1993; Rogers, 1998; Carayannis, 1999), pode-se entender que a TI tem ampliado seu papel na GC, essencialmente no trabalho com o conhecimento explícito (pois o conhecimento tácito, devido a seu próprio conceito, implica restrições a seu manuseio pela TI), equilibrando-se em duas linhas de atuação:

- as tecnologias centradas no indivíduo (human-centric technologies), mais úteis para auxiliar na Internalização do conhecimento explícito e com menos sucesso nas tentativas de transmissão de conhecimentos tácitos (socialização). Sistemas interativos hipertexto e multimídia para a aprendizagem e ferramentas de groupware podem ser mencionados como exemplos destas tecnologias. O foco principal está em facilitar o compartilhamento de interesses e experiências pessoais, devido a um acesso mais dinâmico ao conhecimento explícito;

- as tecnologias centradas na máquina (machine-centric technologies), mais úteis nas tentativas de externalização do conhecimento tácito e no agrupamento dos conhecimentos explícitos (combinação). Envolve sistemas que buscam dinamizar o registro (explícito) de parte do conhecimento (tácito) das pessoas, facilitando, portanto, a externalização e, depois, agrupando este registro junto a inúmeros outros conhecimentos explícitos (realizando então a combinação). Bases de dados, sistemas especialistas, ferramentas de suporte à decisão, agentes de busca na internet etc., são exemplos de tecnologias que podem ser empregadas com esses propósitos. 
Há crescente interdependência entre as tecnologias dessas duas linhas de atuação que caminham em direção a seu emprego de forma integrada nos problemas de GC de uma empresa.

\section{Considerações finais}

As exposições do presente artigo, observadas em conjunto com o que já foi apresentado em Silva (2002), permitem uma relativamente completa visualização de como se aplicar a gestão do conhecimento em empresas. Ressalta aquele artigo a necessária integração com os processos empresariais da organização e suas estratégias competitivas, enquanto neste trabalharam-se as questões de foco mais operacional, relacionadas principalmente com as pessoas envolvidas, as redes de informação e o emprego da tecnologia da informação.

A opção de tratar esse lado operacional pelo detalhamento da consolidada abordagem teórica da criação do conhecimento contribui para tornar mais nítidos e aplicáveis os formatos e conversões do conhecimento, conceitos extremamente relevantes e ainda carentes de mais estudos para seu amplo entendimento e aplicação em modelos de gestão do conhecimento para diferentes tipos de organizações. Um exemplo devidamente comprovado dessa aplicação pode ser encontrado em Silva \& Rozenfeld (2003). No entanto, ainda muitas oportunidades de estudos nessa linha aguardam futuras contribuições de autores da ciência da informação.

Artigo recebido em 22-07-2004 e aceito para publicação de 18 a 21/10/2004.

\section{REFERENNCIAS}

AGOSTA, L. Behind the business intelligence portal. Intelligent Enterprise, v. 2, n. 11, p. 31-38, Aug. 1999.

ALLEN, T. J. Managing the flow of technology: technology transfer and the dissemination of technological information within the $R \& D$ organization. Cambridge, Massachusetts : MIT, 1977.

AMIDON, D. Innovation strategy for the knowledge economy: the Ken Awakening. Boston, Massachusetts : Butterworth-Heinneman, 1997.

ARGYRIS, C.; SCHON, D. Organizational learning: a theory-in-action perspective. Reading, Massachusetts : Addison Wesley, 1978.

BIRCHALL, D.; SMITH, M. Developing the skills of technologists in strategic decision making - a multi-media case approach. International Journal of Technology Management, v. 15, n. 8, p. 854-868, 1998.

BOLISANI, E.; SCARSO, E. Eletronic communication and knowledge transfer. International Journal of Technology Management, v. 20, n. 1-2, p. 116-133, 2000.

BOND, P.; OTTERSON, P. Creativity enhancement software: a systemic approach. International Journal of Technology Management, v. 15, n. 1-2, p. 173-191, Mar./Apr. 1998.
CARAYANNIS, E. G. Fostering synergies between information technology and managerial and organizational cognition: the role of knowledge management. Technovation, v. 19, n. 4, p. 219-231, Apr. 1999.

The strategic management of technological learning in project / program management: the role of extranets, intranets and intelligent agents in knowledge generation, diffusion, and leveraging. Technovation, v. 18, n. 11, p. 697-703, 1998.

CROSS, R.; BAIRD, L. Technology is not enough: improving performance by building organizational memory. Sloan Management Review, v. 41, n. 3, p. 69-78, Spring 2000.

CUMMINGS, A.; OLDHAM, G. Enhancing creativity: managing work contexts for the high potential employee. California Management Review, v. 40, n. 1, p. 22-38, Fall 1997.

DAVENPORT, T. H.; KLAHR, P. Managing customer support knowledge. California Management Review. v. 40, n. 3, p. 195-208, Spring 1998.

; PRUSAK, L. Conhecimento empresarial. Rio de Janeiro : Campus, 1998.

Putting the enterprise into the enterprise system. Harvard Business Review, v. 76, n. 4, p. 121-131, July/Aug. 1998.

; DE LONG, D.W.; BEERS, M.C. Successful knowledge management projects. Sloan Management Review, v. 39, n. 2, p. 43-57, Winter 1998.

et al. Data to knowledge to results: building an analytic capability. California Management Review, v. 43, n. 2, p. 117-138, Winter 2001.

DIENG, I. R. Knowledge management and the internet. IEEE Intelligent Systems $\mathcal{B}$ Their Applications, v. 15, n. 3, p. 14-17, May/June, 2000.

DUTTA, S. Strategies for implementing knowledge-based systems. IEEE Transactions on Engineering Management, v. 44, n. 1, p. 79-90, Feb. 1997.

EDVINSSON, L.; MALONE, M. S. Capital intelectual. São Paulo : Makron Books, 1998.

ELLIOTT, A. Dynamic content is king. Intelligent Enterprise, p. 38-46, Sept. 1999.

GARVIN, D. A. Building a learning organization. Harvard Business Review, v. 71, n. 4, p. 78-91, July/Aug. 1993.

GHOSHAL, S.; NAHAPIET, J. Social capital, intellectual capital, and the organizational advantage. Academy of Management Review, v. 23, n. 2, p. 242-266, Apr. 1998.

GUPTA, A. K.; GOVINDARAJAN, V. Knowledge management's social dimension: lessons from Nucor Steel. Sloan Management Review, v. 42 , n. 1 , p. $77-80$, Fall 2000.

HEIJST, G.; SPEK, R.; KRUIZINGA, E. Corporate memories as a tool for knowledge management. Expert Systems with Applications, v. 10, n. 5, p. 41-54, July, 1997.

KAPLAN, R. S.; NORTON, D. P. A estratégia em ação: balanced scorecard. São Paulo : Campus, 1997.

KLEINER, A.; ROTH, G. How to make experience your company's best teacher. Harvard Business Review, v. 75, n. 5, p. 172-177, Sept./ Oct. 1997.

KLOOSTER, M.; BRINKKEMPER, S.; HARMSEN, F.; WIJERS, G. Intranet facilitated knowledge management: a theory and tool for defining situational methods, Advanced Information Systems Engineering, v. 1250, p. $303-317,1997$. 
Gestão do Conhecimento: uma revisão crítica orientada pela abordagem da criação do conhecimento

KOULOPOULOS, T.; REYNOLDS, H. Enterprise knowledge has a face. Intelligent Enterprise, v. 2, n. 5, p. 29-34, Mar. 1999.

LETHBRIDGE, T. C. Metrics for concept-oriented knowledge bases International. Journal of Software Engineering and Knowledge Engineering, v. 8, n. 2, p. 161-188, June, 1998.

LIEBOWITZ, J.; GILES, P.; GALVIN, T.; HLUCK, G. The role of knowledge-based systems in serving as the integrative mechanism across disciplines. Computers $\mathcal{E}$ Industrial Engineering, v. 34, n. 2, p. 559-564, Apr. 1998.

LIEVROUW, L.; FINN, T.A. New information technologies and informality: comparing organizational information flows using the CSM. International Journal of Technology Management, v. 11, n. 1-2, p. 28 42, 1996.

MARSHALL, L. Facilitating knowledge management and knowledge sharing: new opportunities for information professionals. On Line, v. 21, n. 5, p. 92-98, Sept/Oct. 1997.

MASCITELLI, R. From experience: harnessing tacit knowledge to achieve breakthrough innovation. The Journal of Product Innovation Management, v. 17, n. 3, p. 179-193, May, 2000.

MAURER, H. Web-based knowledge management. Computer, v. 31, n. 3, p. 122-123, Mar. 1998.

MCDERMOTT, R. Why information technology inspired but cannot deliver knowledge management . California Management Review, v. 41, n. 4, p. 103-117, Summer 1999.

MILTON, N.; SHADBOLT, N.; COTTAN, H.; HAMMERSLEY, M. Towards a knowledge technology for knowledge management. International Journal of Human-Computer Studies, v. 51, n. 3, p. 615-641, Sept. 1999.

MOORMAN, C.; MINER, A.S. Organizational improvisation and organizational memory. Academy of Management Review, v. 23, n. 4, p. 698-723, Oct. 1998.

NONAKA, I.; KONNO, N. The concept of 'ba': building a foundation for knowledge creation. California Management Review, v. 40, n. 3, p. 40-54, Spring 1998.

; TAKEUCHI, H. Criação de conhecimento na empresa. Rio de Janeiro : Campus, 1997.

POLANYI, M. The tacit dimension. Glouceste: Peter Smith, 1966.

QUINN, J. B.; BARUCH, J. J.; ZIEN, K. A. Innovation explosion: using intellect and software to revolutionize growth strategies. New York: Free Press, 1997.

ROGERS, E. Enabling innovative thinking: fostering the art of knowledge crafting. International Journal of Technology Management, v. 16, n. 1-3, p. 11-22, May/June, 1998.

RUGGLES III, R. L. Knowledge management tools. Boston, Massachusetts: Butterworth-Heinemann, 1997.

SALERNO, M. Projeto organizacional de produção integrada, flexivel e de gestão democrática: processos, grupos e espaços de comunicaçãonegociação. 1998. 259 f. Tese (Livre-docência) - Departamento de Engenharia de Produção, Escola Politécnica de São Paulo, Universidade de São Paulo, São Paulo, 1998.
SAP. Product lifecycle management: collaborative engineering and project management. [S. 1.], 2000. (SAP white paper).

SCOTT, J.E. Organizational knowledge and the Intranet. Decision Support Systems, v. 23, n. 1, p. 3-17, May. 1998.

SENKER, J. Tacit knowledge and models of innovation. Industrial and Corporate Change, v. 4, n. 2, p. 425-447, 1995.

SILVA, S. L. Informação e competitividade: a contextualização da gestão do conhecimento nos processos organizacionais. Ciência da Informação, v. 31, n. 2, p. 142-151, maio/ago. 2002.

; ROZENFELD, H. Modelo de avaliação da gestão do conhecimento no processo de desenvolvimento do produto: aplicação em um estudo de caso. Produção, v. 13, n. 2, p. 6-20, 2003.

SOLTERO, A. P. Modelo para la representación de una memoria organizacional utilizando herramientas computacionales de Internet. 1997. Dissertação (Maestría en Ciencias con Especialidad en Tecnología Informática) ITESM, Monterrey, México, 1997.

SPEEL, P. H.; ABEN, M. Preserving conceptual structures in design and implementation of industrial KBS. International Journal of Human Computer Studies, v. 49, n. 4, p. 547-575, Oct. 1998.

SPENDER, J. C. Making knowledge the basis of a dynamic theory of the firm. Strategic Management Journal, v. 17, p. 45-62, Dec. 1996. Special issue.

STORCK, J.; HILL, P. A. Knowledge diffusion through strategic communities. Sloan Management Review, v. 41, n. 2, p. 63-74, Winter 2000.

THOMKE, S.; REINERTSEN, D. Agile product development: managing development flexibility in uncertain environments. California Management Review, v. 41, n. 1, p. 8-30, Fall 1998.

TSCHAITSCHIAN, B.; ABECKER, A.; SCHMALHOFER, F. Information tuning with Karat: capitalizing on existing documents. Knowledge Acquisition, Modeling and Management, v. 1319, p. 269-284, 1997.

TUOMI, I. Data is more than knowledge: implications of the reversed knowledge hierarchy for knowledge management and organization memory. Journal of Management Information Systems, v. 16, n. 3, p. 103 117, Winter 1999.

VRIENS, D.; HENDRIKS, P. Knowledge-based systems and knowledge management: friends or foes? Information $\mathcal{E}$ Management, v. 35, p. 113 . 125, 1999.

WENPIN, T. Social capital, strategic relatedness and the formation of intraorganizational linkages. Strategic Management Journal, v. 21, n. 9, Sept. 2000.

WIIG, K. M. Knowledge management: the central management focus for intelligent-acting organizations. Arlington : Schema, 1993. v. 2. 